\title{
Open-Source Direct Simulation Monte Carlo Chemistry Modeling for Hypersonic Flows
}

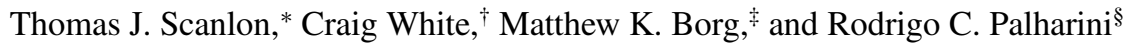 \\ University of Strathclyde, Glasgow G1 1XJ, United Kingdom \\ Erin FarbarI and Iain D. Boyd*** \\ University of Michigan, Ann Arbor, Michigan 48109-2140 \\ Jason M. Reese站 \\ University of Edinburgh, Edinburgh EH9 3HL, United Kingdom \\ and \\ Richard E. Brown章 \\ University of Strathclyde, Glasgow G1 1XJ, United Kingdom \\ DOI: $10.2514 / 1 . J 053370$
}

\begin{abstract}
An open-source implementation of chemistry modeling for the direct simulation Monte Carlo method is presented. Following the recent work of Bird (Bird, G. A., "The Q-K Model for Gas Phase Chemical Reaction Rates," Physics of Fluids, Vol. 23, No. 10, 2011, Paper 106101), an approach known as the quantum-kinetic method has been adopted to describe chemical reactions in a five-species air model using direct simulation Monte Carlo procedures based on microscopic gas information. The quantum-kinetic technique has been implemented within the framework of the dsmcFoam code, a derivative of the open-source computational-fluid-dynamics code OpenFOAM. Results for vibrational relaxation, dissociation, and exchange reaction rates for an adiabatic bath demonstrate the success of the quantum-kinetic model implementation in dsmcFoam when compared with analytical solutions for both inert and reacting conditions. A comparison is also made between the quantum-kinetic and total collision energy chemistry approaches for a hypersonic flow benchmark case.
\end{abstract}

\section{Introduction}

$\mathbf{H}$ YPERSONIC vehicles, which typically operate in rarefied gas environments, are subject to extremes of velocity and altitude. Thus, it is important that the aerodynamic and thermal loads on the vehicle are properly characterized if the feasibility of the vehicle design is to be accurately assessed. The vehicle may also encounter chemical reactions that can have a significant influence on aerodynamic performance and vehicle surface heat flux [1]. Numerical models that fail to incorporate such reacting flows miss an essential part of the flow physics surrounding the vehicle.

The planetary atmospheres through which hypersonic vehicles may pass consist of a number of chemical species, the relative proportion of which varies with altitude. Although the gas in the atmosphere is composed, at the microscopic level, of discrete atoms and molecules, a useful approximation arises if the atmosphere is treated

Received 26 March 2014; revision received 11 October 2014; accepted for publication 30 January 2015; published online 9 April 2015. Copyright $($ C 2014 by the American Institute of Aeronautics and Astronautics, Inc. All rights reserved. Copies of this paper may be made for personal or internal use, on condition that the copier pay the $\$ 10.00$ per-copy fee to the Copyright Clearance Center, Inc., 222 Rosewood Drive, Danvers, MA 01923; include the code $1533-385 \mathrm{X} / 15$ and $\$ 10.00$ in correspondence with the CCC.

*Senior Lecturer, James Weir Fluids Laboratory, Department of Mechanical and Aerospace Engineering; tom.scanlon@strath.ac.uk.

${ }^{\dagger}$ Lecturer, James Weir Fluids Laboratory, Department of Mechanical and Aerospace Engineering; currently School of Engineering, Division of Aerospace Sciences, James Watt South Building, University of Glasgow, Glasgow G12 8QQ, United Kingdom.

${ }^{\ddagger}$ Research Fellow, James Weir Fluids Laboratory, Department of Mechanical and Aerospace Engineering.

${ }^{\S}$ Research Fellow, James Weir Fluids Laboratory, Department of Mechanical and Aerospace Engineering; currently Institute of Aeronautics and Space, Division of Aerodynamics (DCTA/IAE/ALA), Praça Mal. Eduardo Gomes, 50, 12228-904 São José dos Campos, Brazil.

'Research Scientist, Department of Aerospace Engineering.

**Professor, Department of Aerospace Engineering.

†Professor, School of Engineering.

\#Professor, Centre for Future Air-Space Transportation Technology, Department of Mechanical and Aerospace Engineering. as a continuum. The flow over a vehicle moving through the atmosphere can then be modeled by appealing to the fundamental principles of momentum interchange and mass and energy conservation. This continuum approach is at the root of conventional computational fluid dynamics (CFD) methods for the solution of the Navier-Stokes-Fourier (NSF) equations. Indeed, continuum CFD has been applied successfully in many simulations of gas flow around air- and spacecraft and yields good agreement with measured data over a wide range of practically relevant operational conditions [2].

However, continuum-based models have limitations in rarefied gas conditions. The extent of gas rarefaction is traditionally gauged by the Knudsen number $K n$, defined as the ratio of the mean free path of the gas molecules to a characteristic length scale of the vehicle. As the Knudsen number increases, the noncontinuum, particulate-like behavior of the gas becomes more important. Numerical models hoping to simulate such rarefied conditions must be able capture the complex physics shown in Fig. 1 for high-speed vehicle reentry. The flow environment is characterized by a distinct bow shock upstream of the body with a high-temperature region immediately downstream of the shock. In this searingly hot region, chemical reactions may take place involving dissociation, exchange, and ionization, while surface chemistry is also possible. In the wake of the craft, there is a highly rarefied zone within which thermochemical nonequilibrium conditions may exist. The paucity of molecules in this zone may necessitate the region being described using a noncontinuum, particle-based formulation.

It may be possible to describe such complex flow conditions using the Boltzmann equation, which is appropriate for the entire range of Knudsen numbers likely to be encountered by hypersonic vehicles. However, the Boltzmann equation is extremely difficult to solve numerically due to its high dimensionality and the complexity of the collision term. Simplification of the collision term in the equation through the Bhatnagar-Gross-Krook approximation [3] has given rise to a number of new numerical algorithms, such as the model Boltzmann equation solver developed by Yang and Huang [4]; however, these nascent techniques have yet to establish themselves as practical simulation tools, particularly for high-speed reacting flows.

The problem that the conventional Navier-Stokes-Fourier (NSF) equations have in capturing rarefaction is highlighted in Fig. 2 [5] . 


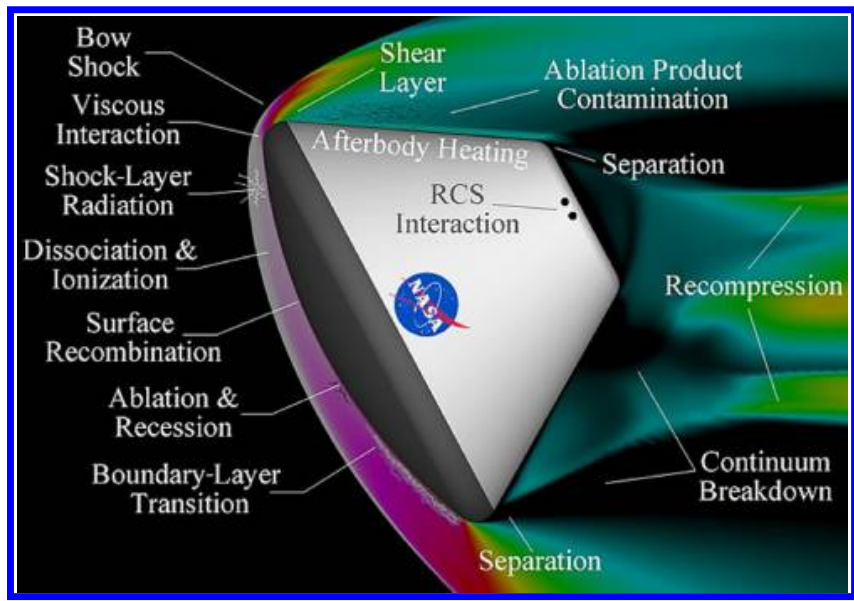

Fig. 1 Complex physics in planetary vehicle reentry (image credit NASA).

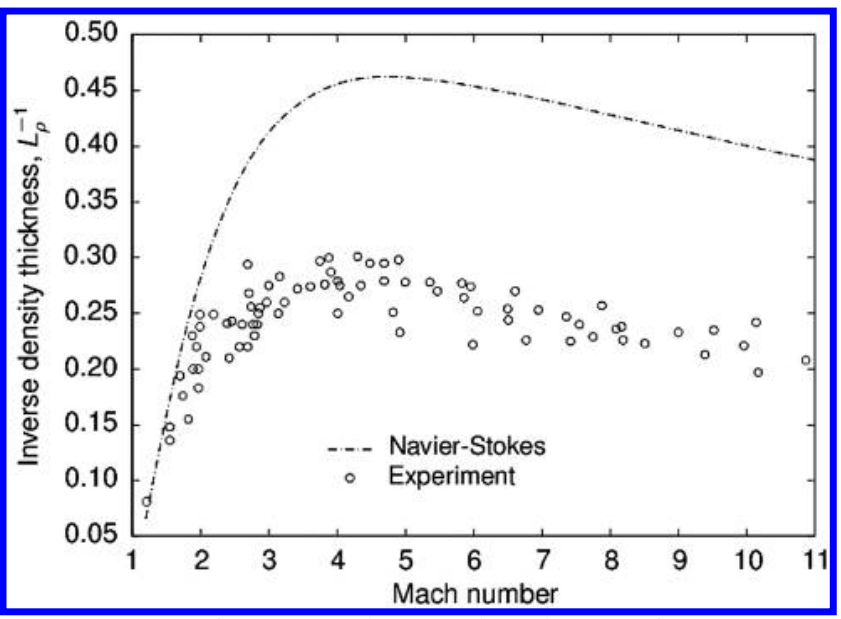

Fig. 2 Inverse of the normalized density thickness of normal shock waves in argon gas up to Mach 11.

The normal shock wave is a fundamental component of many high-speed aerodynamic flows (e.g., in the central part of the bow shock). Rarefaction causes the shock to be relatively thick (of the order of a few gas mean-free-paths, of the same order as the stand-off distance of the bow shock from the vehicle leading edge). This figure shows that the NSF equations consistently predict shocks in argon gas that are some $50 \%$ thinner than observed experimentally. That the fluid dynamic equations have such difficulty in predicting the behavior in such a simple flow case calls their appropriateness for more complex rarefied flow fields into question.

Noncontinuum behavior can be accommodated to some extent in conventional NSF approaches to modeling the gas dynamics around aerospace vehicles, for instance by incorporating a finite slip velocity between the gas and any solid surfaces that are immersed within the flow. A computationally efficient gas flow method, but one that has had only modest success to date, is to establish either a $K n$ series or a Hermite polynomial approximation to the molecular velocity distribution function in the Boltzmann equation. To first order (i.e., for near-equilibrium flows) this approach yields the NSF set, but the solution method can be continued to second and higher orders to incorporate more and more of the salient characteristics of a rarefied flow. This family of so-called extended hydrodynamic equations has various different members, including the Burnett, Grad 13-moment, R13, R26 equations, and others. Generally, they all have great difficulty in achieving stable physical solutions of high-Mach-number flows. Their nonlinearity also makes them difficult to solve numerically and, because they are higher-order in the gradients of flow properties, their solution requires additional boundary conditions that are not easy to define. For these reasons, extended hydrodynamics has not established a firm place in the armory of tools a high-speed aerodynamicist can deploy.

In highly rarefied environments $(K n>0.1)$, accommodation of the thermochemical nonequilibrium effects that occur in the flow away from surfaces remains a challenging problem. For this reason, analysis of gas flows in the noncontinuum regime is most naturally conducted using specialized computational techniques that are derived from a statistical mechanical representation of the behavior of the individual particles comprising the flow. The most successful of these techniques is undoubtedly the direct simulation Monte Carlo (DSMC) approach, originally proposed by Bird [6].

The DSMC technique instructs particles to move and collide using kinetic-theory considerations that can capture the noncontinuum gas behavior accurately. DSMC considers molecular collisions using stochastic rather than deterministic procedures over a time step that is a small fraction of the mean collision time, and each DSMC particle represents a large number of real gas molecules. The decoupling of particle ballistic motion and particle collisions improves the computational efficiency of DSMC greatly in comparison with other particle methods such as molecular dynamics. The computational domain is divided into either a structured or unstructured grid of cells, with each cell of a dimension that is a small fraction of the local mean free path. The cells are then used to select particles for collisions on a probabilistic basis and are used for sampling the macroscopic flow properties. Intermolecular collisions are handled probabilistically using phenomenological models that are designed to reproduce real fluid behavior when the flow is examined at the macroscopic level. The DSMC technique has been shown to provide a solution to the Boltzmann equation as the number of simulated particles tends toward infinity [7]. The DSMC approach is currently the dominant numerical method for rarefied gas flow applications.

For engineering purposes, the gas in the Earth's atmosphere may be assumed to be a binary mixture of oxygen $\left(\mathrm{O}_{2}\right)$ and nitrogen $\left(\mathrm{N}_{2}\right)$. In the DSMC methodology, particle clusters must be endowed with the correct properties to capture kinetic and rotational modes of energy storage. Vibrational excitation of the gas molecules as well as dissociation of both oxygen and nitrogen are likely to be important features of the flow around any hypersonic vehicle at the highest altitudes (80-120 km) and speeds, whereas even at lower speeds and altitudes, vibrational excitation and limited dissociation of oxygen are still likely to be important [8]. Such real-gas effects need to be properly accounted for. The DSMC technique normally models air as either a five-species mixture using dissociation, recombination, and exchange reactions or as a mixture of 11 -species including ionization. In conventional CFD, reaction rates are calculated according to the Arrhenius law [8]. However, this relies on data sourced from equilibrium conditions, which may be inappropriate for rarefied hypersonic flows. In contrast, the DSMC method, with its particulate approach, is able to capture successfully the thermochemical nonequilibrium effects encountered in high-speed rarefied gas environments [1,9].

The goal of this work is to describe, verify, and discuss the implementation of a state-of-the-art chemistry model, using the quantum-kinetic (Q-K) approach [9], in an open-source, nonproprietary DSMC code for exploitation by the scientific community.

\section{Chemistry Modeling in dsmcFoam}

The DSMC code used for this paper is called dsmcFoam. The code has been written within the framework of the open-source $\mathrm{C}++\mathrm{CFD}$ toolbox OpenFOAM [10]. The main features of the dsmcFoam code include the capability to perform both steady and transient DSMC simulations for multispecies conditions to model arbitrary twodimensional/three-dimensional geometries using unstructured polyhedral meshes and unlimited parallel processing. The original version of dsmcFoam determines intermolecular collisions for polyatomic species using the variable hard-sphere (VHS) model and applies the phenomenological Larsen-Borgnakke model to distribute postcollision energy between the translational and rotational modes [6]. A series of successful benchmark trials have been carried out to verify the dsmcFoam code for nonreacting flows [11]. 


\section{A. Vibrational Energy and the Quantum-Kinetic Chemistry Model}

The vibrational energy mode plays a key role in chemical reactions using the Q-K model. This mode was excluded in previous versions of dsmcFoam [11], and so this section describes the implementation and validation of the vibrational mode into the code. The vibrational modes of a gas are generally active when the system is sufficiently energized (e.g., under the high-enthalpy conditions commonly found in hypersonic applications). The vibrational mode forms part of the total energy budget and limits the amount of post-collision energy available to the translational and rotational modes. In addition, it often introduces a new mode of nonequilibrium to a rarefied gas system because the number of collisions required for vibrational relaxation is significantly higher than that for translational or rotational equilibration.

For the implementation of the vibrational mode in dsmcFoam, it is proposed that the vibrational energy can only assume discrete quantum values, as described by Haas et al. [12] and Bergman and Boyd [13]. We consider a serial application of the quantum LarsenBorgnakke method using the harmonic oscillator model to redistribute vibrational energy before rotational and translational energy exchange are considered. It is desirable in DSMC to avoid the use of the macroscopic temperature whenever possible because colliding particles have no knowledge of the surrounding temperature. Here, we discuss a model for the redistribution of vibrational energy based on the collision energy (as opposed to the local macroscopic temperature of the gas). The first step is to define a "quantized collision temperature", $T_{\text {coll }}$, based on the collision energy of a particle pair $p$ and $q[14]$ :

$$
T_{\mathrm{coll}}=\frac{i_{\max } \theta_{v}}{7 / 2-\omega}
$$

where

$$
i_{\max }=\left\lfloor\frac{E_{c}}{k \theta_{v}}\right\rfloor
$$

and $\lfloor\ldots\rfloor$ denotes truncation; $i_{\max }$ is the maximum quantum level available to the molecule; $\theta_{v}$ is the vibrational temperature; $k$ is the Boltzmann constant; $\omega$ is the temperature exponent of viscosity; and $E_{c}$ is the sum of the relative translational energy of the collision pair and the precollision vibrational energy of the molecule under consideration, i.e.,

$$
E_{c}=\varepsilon_{\mathrm{tr}, p q}+\varepsilon_{v, p}
$$

The vibrational collision number $Z_{v}$ can then be calculated as [14]

$$
Z_{v}=\left(\frac{\theta_{d}}{T_{\text {coll }}}\right)^{\omega}\left[Z_{\mathrm{ref}}\left(\frac{\theta_{d}}{T_{Z_{\mathrm{ref}}}}\right)^{-\omega}\right]^{\left[\left(\theta_{d} / T_{\mathrm{coll}}\right)^{1 / 3}-1\right] /\left[\left(\theta_{d} / T_{Z_{\mathrm{ref}}}\right)^{1 / 3}-1\right]}
$$

where $\theta_{d}$ is the characteristic dissociation temperature, and $Z_{\text {ref }}$ is the vibrational collision number at a reference temperature $T_{Z_{\text {ref }}}$, which is usually taken to be the characteristic vibrational temperature $\theta_{v}$, such that [15]

$$
Z_{\text {ref }}=\left(\frac{C_{1}}{T_{Z_{\text {ref }}^{\omega}}^{\omega}}\right) \exp \left(C_{2} T_{Z_{\text {ref }}}^{-1 / 3}\right)
$$

, where $C_{1}$ and $C_{2}$ are constants that can be found in Appendix A of [6], and $T_{Z_{\text {ref }}}$ is set as $\theta_{v}$.

Once the vibrational collision number has been calculated, the particle is tested for vibrational energy exchange and is accepted if

$$
\frac{1}{Z_{v}}>R_{f}
$$

where $R_{f}$ is a random number between 0 and 1 . An integer postcollision vibrational quantum level $i^{*}$ is chosen uniformly between 0 and the maximum possible level $i_{\max }^{*}$, and the acceptance-rejection method is used to select a value of $i^{*}$ using a quantized version of the Larsen-Borgnakke probability ratio [13]:

$$
\frac{P}{P_{\max }}=\left(1-\frac{i^{*} k \theta_{v}}{E_{c}}\right)
$$

The total energy of the pair is reduced accordingly, and particle $p$ is then considered for relaxation into the rotational and translational modes using the standard Larsen-Borgnakke approach.

To verify the implementation of this vibrational energy exchange, a test case involving relaxation to equilibrium is considered. This test case involves an adiabatic box filled with nitrogen gas, in a manner similar to that considered by Bird [16]. The translational and rotational temperatures are initially $20,0 \overline{00} \mathrm{~K}$, and there is no energy in the vibrational mode. The reference vibrational collision number $Z_{\text {ref }}$ is 19.8 at a reference temperature of $20,000 \mathrm{~K}$. The dsmcFoam simulation is performed using the VHS collision model, with the following properties ascertained at $T_{\text {ref }}=273 \mathrm{~K}, m=46.5 \times 10^{-27} \mathrm{~kg}$, $\omega=0.74$, and $d_{\text {ref }}=4.17 \times 10^{-10} \mathrm{~m}$, where $d_{\text {ref }}$ and $m$ are the molecular diameter and mass, respectively.

The cell is filled with 1.2 million DSMC particles, and as the particles begin to collide, energy is transferred to the vibrational mode, and equipartition is achieved at a temperature of around 14,720 K after a period of relaxation, as shown in Fig. 3 . The "collision number" on the abscissa is calculated as the product of the instantaneous collision rate from the simulation and the physical time that has elapsed. The dsmcFoam results agree well with those of Bird's DS2V code [16]. The rotational temperature follows the translational temperature closely, and the vibrational temperature takes a longer time to arrive at equilibrium. The relaxation rates of all temperatures and the final equilibrium temperature are in good agreement with Bird's results.

\section{B. Quantum-Kinetic Chemical Reactions}

Until recently, chemically reacting gas flows in DSMC have relied upon input data derived at the macroscopic level and reaction rate coefficients that are derived from equilibrium theory. This method for treating chemical reactions was introduced by Bird in 1979 and is known as the total collision energy (TCE) model [17]. The TCE chemical reaction model is well established and is used in mature DSMC codes such as MONACO [18].

As an alternative approach, Bird has recently proposed a chemical reaction model that is founded on the quantum Larsen-Borgnakke method described in Sec. II.A. This model has only a limited dependence on macroscopic data because the vibrational collision number is a function of the collision temperature (microscopic level) and a reference temperature (macroscopic level). The method also does not require that the gas be in a state near thermodynamic equilibrium. This is termed the quantum-kinetic (Q-K) model, and it has been developed over the past five years $[\underline{9}, \underline{1}, \underline{14}, \underline{19}]$.

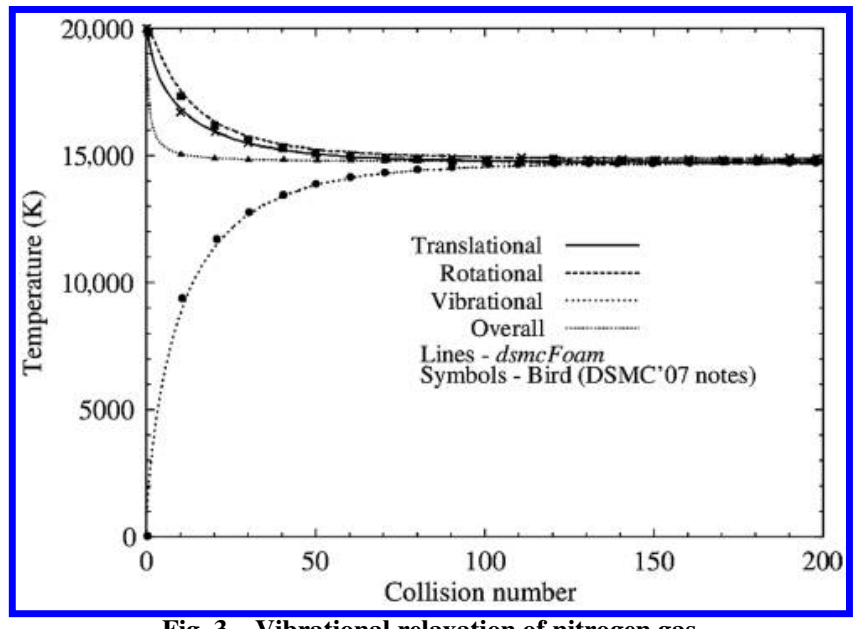

Fig. 3 Vibrational relaxation of nitrogen gas. 
In this paper, we consider dissociation and exchange reactions using the Q-K approach with the aim of creating a five-species (oxygen $\mathrm{O}_{2}$, nitrogen $\mathrm{N}_{2}$, nitric oxide $\mathrm{NO}$, atomic oxygen $\mathrm{O}$, and atomic nitrogen $\mathrm{N})$ air chemistry model for use in the open-source dsmcFoam code.

\section{Dissociation Reactions}

The condition for a dissociation reaction in the $\mathrm{Q}-\mathrm{K}$ model is as follows: if the energy in a collision is high enough to allow a dissociation event, it will always occur. The reaction being considered is $A B+C \rightarrow A+B+C$, where $A B$ is the molecule considered for dissociation, $C$ is the reactant partner (either a molecule or an atom), and $A$ and $B$ are the atomic products of the dissociation. Considering the serial application of the quantum Larsen-Borgnakke model, the collision energy $E_{c}$ of a particle pair $(A B+C)$ is the sum of the relative translational energy and the precollision vibrational energy of the particle currently being considered, as described by Eq. (3). The maximum vibrational level that can be selected $i_{\max }$ is given by Eq. (2). If $i_{\max }$ is beyond the dissociation limit, i.e.,

$$
i_{\max }>\frac{\theta_{d}}{\theta_{v}}
$$

, then the molecule $A B$ must be dissociated before any vibrational or rotational relaxation can be considered.

A distinct feature of the Q-K model is that, although the DSMC implementation does not necessitate that the gas be in equilibrium, if it is assumed that it is, then analytical solutions for the reaction rates can be derived. For a VHS gas in equilibrium, the rate coefficient $k_{f}(T)$ for the dissociation of a molecule $A B$ with a molecule or atom $C$ is

$$
k_{f}(T)=R_{\text {coll }}^{A B, C} \gamma\left(i_{\max }\right)^{A B, C}
$$

where $R_{\text {coll }}^{A B, C}$ is the collision rate between species $A B$ and $C$ divided by the number density product. For an equilibrium VHS gas, this is

$$
R_{\text {coll }}^{A B, C}=\frac{2 \pi^{1 / 2}}{\alpha}\left(r_{\text {ref }}^{A B}+r_{\text {ref }}^{C}\right)^{2}\left(\frac{T}{T_{\text {ref }}}\right)^{1-\omega^{A B, C}}\left(\frac{2 k T_{\text {ref }}}{m_{r}^{A B, C}}\right)^{1 / 2}
$$

where $r_{\text {ref }}, T_{\text {ref }}$, and $\omega$ are the standard VHS properties of the relevant gas species; $m_{r}$ is the reduced mass of the collision pair; and $T$ is the macroscopic temperature of the gas. The symmetry factor $\alpha$ is set to 2 if the molecules in the collision are identical or 1 for dissimilar molecules. The $\gamma\left(i_{\max }\right)^{A B, C}$ parameter defines the fraction of collisions that will have sufficient energy to dissociate. Taking $i$ as the precollision vibrational state of the dissociating molecule, the result is

$$
\begin{aligned}
& \gamma\left(i_{\max }\right)^{A B, C}=\sum_{i=0}^{i_{\max }-1} \frac{1}{z_{v}(T)^{A B}} \\
& \times\left[Q\left(\frac{5}{2}-\omega^{A B, C},\left(\frac{\left(i_{\max }-i\right) \theta_{v}^{A B}}{T}\right)\right) \exp \left(\frac{-i \theta_{v}^{A B}}{T}\right)\right]
\end{aligned}
$$

where $Q(a, x)=\Gamma(a, x) / \Gamma(a)$ is a form of the incomplete Gamma function, and $z_{v}(T)^{A B}=1 /\left[1-\exp \left(-\theta_{v} / T\right)\right]$ is the vibrational partition function [9].

\section{Dissociation in dsmcFoam}

We have implemented Q-K dissociation reactions in dsmcFoam for the following nine molecule-molecule dissociations, which we term "type I" dissociations:

$$
\mathrm{O}_{2}+\mathrm{O}_{2} \rightarrow \mathrm{O}+\mathrm{O}+\mathrm{O}_{2}
$$

$$
\mathrm{N}_{2}+\mathrm{N}_{2} \rightarrow \mathrm{N}+\mathrm{N}+\mathrm{N}_{2}
$$

$$
\begin{aligned}
& \mathrm{NO}+\mathrm{NO} \rightarrow \mathrm{N}+\mathrm{O}+\mathrm{NO} \\
& \mathrm{O}_{2}+\mathrm{N}_{2} \rightarrow \mathrm{O}+\mathrm{O}+\mathrm{N}_{2} \\
& \mathrm{~N}_{2}+\mathrm{O}_{2} \rightarrow \mathrm{N}+\mathrm{N}+\mathrm{O}_{2} \\
& \mathrm{O}_{2}+\mathrm{NO} \rightarrow \mathrm{O}+\mathrm{O}+\mathrm{NO} \\
& \mathrm{N}_{2}+\mathrm{NO} \rightarrow \mathrm{N}+\mathrm{N}+\mathrm{NO} \\
& \mathrm{NO}+\mathrm{O}_{2} \rightarrow \mathrm{N}+\mathrm{O}+\mathrm{O}_{2}
\end{aligned}
$$

$$
\mathrm{NO}+\mathrm{N}_{2} \rightarrow \mathrm{N}+\mathrm{O}+\mathrm{N}_{2}
$$

and the following six molecule-atom dissociations, or "type II" dissociations:

$$
\begin{aligned}
& \mathrm{O}_{2}+\mathrm{O} \rightarrow \mathrm{O}+\mathrm{O}+\mathrm{O} \\
& \mathrm{O}_{2}+\mathrm{N} \rightarrow \mathrm{O}+\mathrm{O}+\mathrm{N}
\end{aligned}
$$

$$
\mathrm{N}_{2}+\mathrm{O} \rightarrow \mathrm{N}+\mathrm{N}+\mathrm{O}
$$

$$
\mathrm{N}_{2}+\mathrm{N} \rightarrow \mathrm{N}+\mathrm{N}+\mathrm{N}
$$

$$
\mathrm{NO}+\mathrm{O} \rightarrow \mathrm{N}+\mathrm{O}+\mathrm{O}
$$

$$
\mathrm{NO}+\mathrm{N} \rightarrow \mathrm{N}+\mathrm{O}+\mathrm{N}
$$

We consider dissociation reactions for two cases involving either thermochemical equilibrium or nonequilibrium. For the case of equilibrium, if a DSMC particle meets the criterion for dissociation to occur [Eq. (8)], then we may simply update a counter within our DSMC simulation during which no particle splitting takes place. This procedure enables reaction rates to be calculated at a constant temperature for a gas in equilibrium and then compared to the analytical Q-K solution of Eq. (9). In his 2011 Q-K paper [9], Bird does not present any DSMC simulation data; it is solely analytical work with a description of the DSMC procedure for each reaction. Gallis et al. [1] and Wysong et al. [19] have presented some DSMC simulation data; however, this work was performed using a DSMC implementation for exchange reactions that has since been superseded.

For the nonequilibrium case, particle splitting may occur, and nascent species are introduced with the associated conservation of mass, momentum, and energy. This procedure gives rise to an initial nonequilibrium mixture, which eventually settles down to steadystate, thermochemical equilibrium. For the nonequilibrium case, the results from the dsmcFoam Q-K implementation are compared with analytical solutions. The analytical results are generated for the simultaneous solution of rate kinetic equations describing the time evolution of the concentration of each chemical species as well as the 


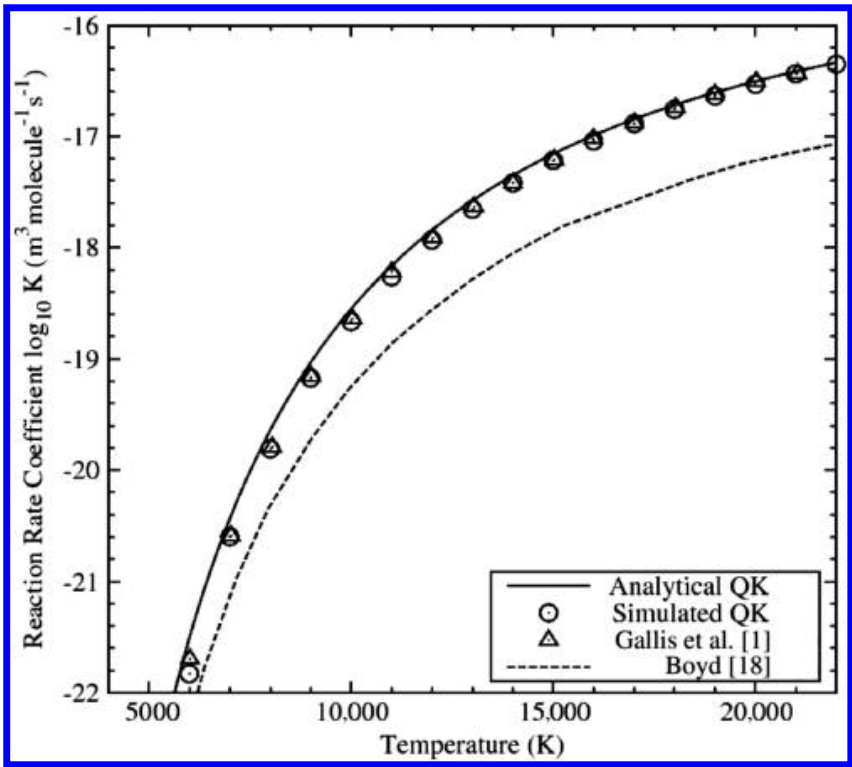

Fig. 4 Equilibrium dissociation rate coefficient for type I dissociation, $\mathbf{N}_{2}+\mathbf{N}_{2} \rightarrow \mathbf{N}+\mathbf{N}+\mathbf{N}_{2}$.

equilibrium temperature of the reacting gas mixture [20]. The overall rate of change of the concentration of species $s$ is given by the summation over all individual rate processes of the form

$$
\frac{\mathrm{d}\left[X_{s}\right]}{\mathrm{d} t}=-k_{f}\left[X_{s}\right]\left[X_{r}\right]+k_{b}\left[X_{p 1}\right]\left[X_{p 2}\right]
$$

where $k_{f}$ and $k_{b}$ are forward and backward rate coefficients, $X_{r}$ is a reactant species, and $X_{p 1}$ and $X_{p 2}$ are product species. The forward reaction only is considered, and backward recombination is deactivated. The $k_{f}$ is determined from the equilibrium Q-K rate [Eq. (9)] and is set in Arrhenius form. A system of five equations is solved simultaneously for species $\mathrm{N}_{2}, \mathrm{O}_{2}, \mathrm{NO}, \mathrm{N}$, and $\mathrm{O}$ for hightemperature air. The overall energy balance is also analyzed accounting for the internal energy modes (translation, rotation, vibration) and chemical reactions to determine the equilibrium temperature.

\section{Equilibrium Dissociation}

Adiabatic box simulations are performed to measure the equilibrium dissociation rate coefficients for each reaction. A single cubic cell of side length $1 \times 10^{-5} \mathrm{~m}$, with six specularly reflective surfaces, was used for this purpose. Following the work of Haas and McDonald [21], a total of 50,000 DSMC particles were used in each simulation, a time step size of $1.52 \times 10^{-9} \mathrm{~s}$ was adopted, and the rotational and vibrational collision numbers were set to 1 . The particles were allowed to move and exchange energy, but no particle splitting occurred. Only the forward reaction was considered during the reaction, and recombination was discounted.

Figure 4 shows the equilibrium reaction rate coefficients for the type I dissociation reaction defined by Eq. (13), i.e., dissociation of a nitrogen molecule in a collision with another nitrogen molecule. The analytical and numerical Q-K solutions are in satisfactory agreement, whereas the dsmcFoam $Q-K$ values remain in excellent agreement with the DSMC results of Gallis et al. [1], thus verifying the dsmcFoam implementation of the Q-K approach for dissociation. In comparison with the TCE results of Boyd [18], the Q-K values show a similar trend; however, there is a discordance in the level of agreement, and higher Q-K values are predicted.

Although not presented in this paper for reasons of conciseness, the remaining type I and type II dissociation reactions have been implemented in dsmcFoam, and these demonstrate similar levels of agreement to those shown in Fig. 4 when compared with the Q-K analytical rates, the Gallis et al. [1] DSMC results, and Boyd's [1] TCE rates.

\section{Exchange Reactions}

An exchange reaction comprises one stable molecule and one radical before and after the reaction occurs. These reactions take the form $A+B \leftrightarrow C+D$, where $A$ and $C$ are molecules, and $B$ and $D$ are atoms. In the five-species air model considered in this paper, there are two sets of exchange reactions:

$$
\mathrm{N}_{2}+\mathrm{O} \leftrightarrow \mathrm{NO}+\mathrm{N}
$$

and

$$
\mathrm{NO}+\mathrm{O} \leftrightarrow \mathrm{O}_{2}+\mathrm{N}
$$

Each of these has a forward (endothermic) and a backward (exothermic) direction, resulting in a total of four exchange reactions. The DSMC implementation of exchange chemistry leads to reactions being possible if the collision energy $E_{c}$ is greater than the activation energy $E_{a}$, with a probability of

$$
P=\left(1-\frac{E_{a}}{E_{c}}\right)^{3 / 2-\omega} / \sum_{i=0}^{i_{\max }}\left(1-\frac{i k \theta_{v}}{E_{c}}\right)^{3 / 2-\omega}
$$

The summation in the denominator can be taken as unity when $E_{a} / k<\theta_{v}$, and the analytical Q-K rates given by Bird [9] are

$$
k_{f}(T)=R_{\text {coll }}^{A, B} \exp \left(\frac{E_{a}^{A, B}}{k T}\right) / z_{v}(T)^{A}
$$

and

$$
k_{r}(T)=R_{\text {coll }}^{C, D} \exp \left(\frac{E_{a}^{C, D}}{k T}\right) / z_{v}(T)^{C}
$$

where $k_{f}(T)$ and $k_{r}(T)$ are the forward and reverse reaction rates, respectively; $z_{v}(T)$ is the vibrational partition function; and $R_{\text {coll }}$ is the collision rate between the species indicated in the superscript and is given by Eq. (10).

The default activation energy for the forward exchange reactions is the heat of reaction, $E_{r}$, and for the reverse reaction, it is zero.

To ensure that the ratio of the number of forward to reverse exchange reactions is consistent with that predicted by statistical mechanics, it is necessary to adjust the activation energies in a phenomenological manner. For forward exchange reactions, the adjusted activation energy $E_{a, f}^{\prime}$ is

$$
E_{a, f}^{\prime}=\left|E_{h}\right|\left[1+a\left(\frac{T}{273}\right)^{b}\right]
$$

and for the reverse exchange reaction, it is

$$
E_{a, r}^{\prime}=\left|E_{h}\right|\left[a\left(\frac{T}{273}\right)^{b}\right]
$$

where $\left|E_{h}\right|$ is the modulus of the heat of formation, and $a$ and $b$ are adjustable parameters.

In our dsmcFoam implementation, we have chosen values of $a$ and $b$ for the four exchange reactions that enable our DSMC results to be in satisfactory agreement with both the analytical equilibrium Q-K rates of Eqs. (31) and (32) and the analytical nonequilibrium rates predicted by Eq. (27), and, finally, to ensure that the ratio of the forward to reverse reactions is consistent with that predicted by statistical mechanics [9]. The parameters $a$ and $b$ are given in Table 1 and were determined using Bird's QKRates program [22]. Figure $\overline{5}$ shows the ratio of forward to reverse exchange reaction rate for $\mathrm{N}_{2}+\mathrm{O} \leftrightarrow \mathrm{NO}+\mathrm{N}$, and reasonable agreement is found in comparison with the equilibrium constant from statistical mechanics [22].

It should be noted that the equilibrium parameters $a$ and $b$ may be chosen such that both curves in Fig. $\underline{5}$ are identical; however, 
Table 1 Parameters for adjusted activation energies in Eqs. (33) and (34)

\begin{tabular}{lll}
\hline \hline Exchange reaction & \multicolumn{1}{c}{$a$} & \multicolumn{1}{c}{$b$} \\
\hline $\mathrm{NO}+\mathrm{O} \rightarrow \mathrm{O}_{2}+\mathrm{N}$ & 0.085 & 0.65 \\
$\mathrm{O}_{2}+\mathrm{N} \rightarrow \mathrm{NO}+\mathrm{O}$ & 0.1 & 0.1 \\
$\mathrm{~N}_{2}+\mathrm{O} \rightarrow \mathrm{NO}+\mathrm{N}$ & 0.15 & 0.15 \\
$\mathrm{NO}+\mathrm{N} \rightarrow \mathrm{N}_{2}+\mathrm{O}$ & 0.033 & 0.8 \\
\hline \hline
\end{tabular}

compromise values were selected to obtain optimum agreement also with Eqs. (27), (31), and (32) as explained previously.

Adiabatic box simulations are performed to measure the equilibrium rate coefficients for two of the exchange reactions shown in Table 1. The cases have set-up parameters identical to those for the equilibrium dissociation reactions presented in Sec. II.C.2, and a 50\% split of each species by number is used as the initial conditions.

Figure 6 shows the equilibrium reaction rate for the forward endothermic exchange reaction $\mathrm{NO}+\mathrm{O} \rightarrow \mathrm{O}_{2}+\mathrm{N}$. The TCE rate from [18], the DSMC data from Gallis et al. [1], and a rate representative of the National Institute of Standards and Technology (NIST) database [23] are also included. In addition to these, the dsmcFoam implementation for exchange has been calculated using two different definitions for temperature in Eqs. (33) and (34), with these being the macroscopic and the collision temperature. The collision temperature has been used for all dsmcFoam calculations in this paper, and its definition is [9]

$$
T_{\text {coll }}=\left(m_{r}^{A, B} c_{r}^{2} /(2 k)\right) /\left(5 / 2-\omega^{A, B}\right)
$$

In his 2011 paper, Bird [9] recommends that, if the collision temperature is to be used, then the exchange factor $a$ in Eqs. (33) and (34) needs to be replaced by

$$
a^{\prime}=\left[\left(5 / 2-\omega^{A, B}\right)^{b} \Gamma\left(5 / 2-\omega^{A, B}\right) / \Gamma\left(5 / 2-\omega^{A, B}+b\right)\right] a
$$

This step was considered necessary due to the inequality of macroscopic and collision temperatures when the parameter $b$ is not equal to zero or unity. However, our dsmcFoam calculations for exchange showed no apparent difference whether $T_{\text {coll }}$ was used in tandem with Eq. (36) or not. It is desirable that the collision temperature be used in DSMC procedures rather than the macroscopic value because information should be passed upward from the molecular level.

In any case, it is clear from all our figures for exchange reactions that the use of macroscopic temperature in our dsmcFoam imple-

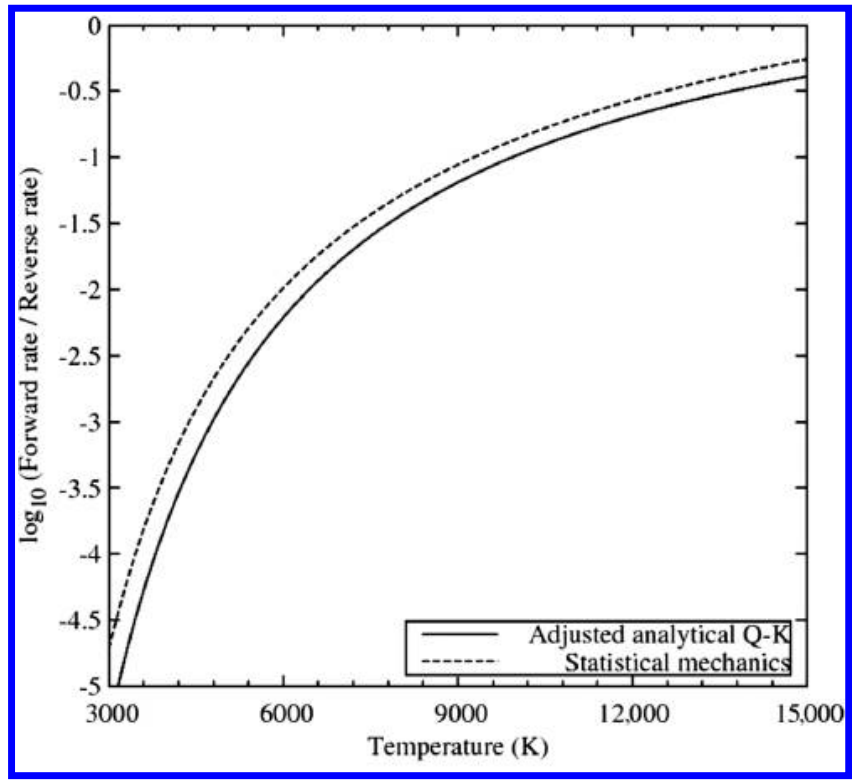

Fig. 5 Forward to reverse rate ratio for $\mathrm{N}_{2}+\mathrm{O} \leftrightarrow \mathrm{NO}+\mathrm{N}$. mentation produces equilibrium rates that match the $\mathrm{Q}-\mathrm{K}$ analytical values. Such a result helps verify our coding; however, the application of the collision temperature for exchange does produce results that are highly sensitive to the choice of the exponent parameter $b$ in Eqs. (33) and (34). Averaging over all collisions, $\bar{T}_{\text {coll }}=\bar{T}_{\text {macro }}$; however, $\bar{T}_{\text {coll }}^{b}=T_{\text {macro }}^{b}$ unless $b=1$ or 0 (note that the overbar represents average quantities). This can explain why there is closer agreement between the exchange rates found using $T_{\text {coll }}$ and $T_{\text {macro }}$ in Fig. 7, as they use small values of the exponent $b$, whereas there is an increased disparity between the $T_{\text {coll }}$ and $T_{\text {macro }}$ rates in Fig. $\underline{6}$, and a larger value of $b$ is employed.

The simulated Q-K rates using $T_{\text {coll }}$ are seen in Fig. $\underline{6}$ to fall within the range covered by the TCE and the NIST rates for temperatures in excess of around $7000 \mathrm{~K}$. It is interesting to note that the Gallis et al. [1] data, based on a DSMC implementation, which did not consider the adjustment of activation energies, show consistently higher reaction rates across the range of temperatures considered.

The corresponding reverse exothermic equilibrium rate for the $\mathrm{O}_{2}+\mathrm{N} \rightarrow \mathrm{NO}+\mathrm{O}$ exchange reaction is shown in Fig. 7. Additional experimental data from Gupta et al. [24] and Bortner [25] have been

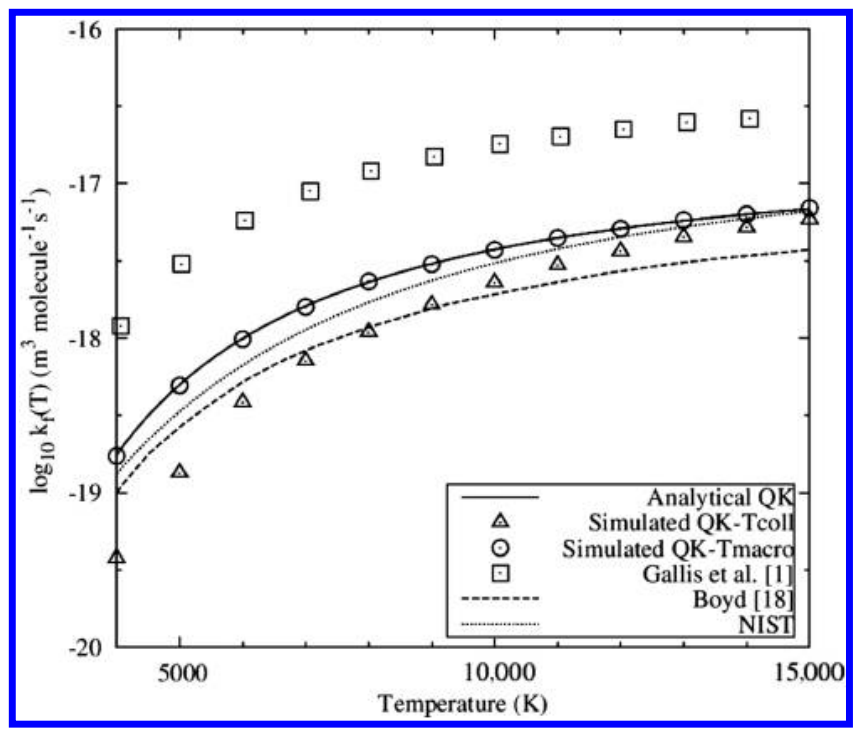

Fig. 6 Forward endothermic equilibrium exchange rate coefficient for $\mathrm{NO}+\mathrm{O} \rightarrow \mathrm{O}_{2}+\mathrm{N}$.

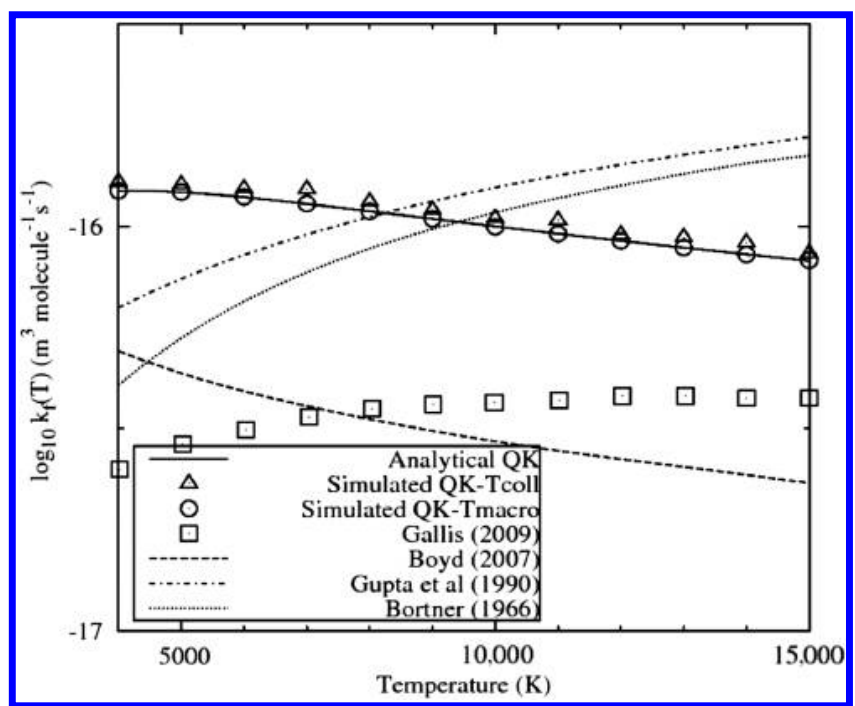

Fig. 7 Reverse exothermic equilibrium exchange rate coefficient for $\mathrm{O}_{2}+\mathrm{N} \rightarrow \mathrm{NO}+\mathrm{O}$. 
included. Close agreement is observed between the Q-K rates predicted by the collision and macroscopic temperatures because the coefficient $b$ is small $(0.1)$ for this reaction. The trend of decreasing reaction rate with temperature is similar for both Q-K and TCE; however, the Q-K rates are higher across the range of temperatures studied and are closer to the results of Gupta et al. and Bortner. In comparison with the activation energy-adjusted Q-K approach presented in this paper, the previous incarnation of Q-K considered by Gallis et al. [1] predicts lower rates for this reaction, with their results being closer to the TCE rates. A similar level of agreement was found for the forward and reverse equilibrium rates for the $\mathrm{N}_{2}+\mathrm{O} \rightarrow$ $\mathrm{NO}+\mathrm{N}$ reaction.

\section{E. Nonequilibrium Dissociation and Exchange in Air}

Unlike the equilibrium rate calculations shown previously, the nonequilibrium dissociation and exchange reactions presented in this section involve particle splitting. The physical dissociation or exchange of the molecules is accompanied by a process of detailed balance for mass, momentum, and energy as DSMC particles disintegrate or exchange.

The complete set of 19 reactions shown in Table 2 is considered in an adiabatic box filled with air at 0.063 atm pressure at an initial composition of $79 \% \mathrm{~N}_{2}$ and $21 \% \mathrm{O}_{2}$ and temperature of $30,000 \mathrm{~K}$. Other simulation parameters are identical to those described in Sec. II.C.2. A single-cell adiabatic cube of side length $1 \times 10^{-5} \mathrm{~m}$ is again employed, and 50,000 initial DSMC particles were used with a time step of $1.52 \times 10^{-9} \mathrm{~s}$. The fate of each species is measured in a transient manner as the reaction proceeds from equilibrium initial conditions through a nonequilibrium reaction process to a final state approaching equilibrium. Once again, the backward recombination reaction has been deactivated, and only the forward chemistry is dealt with. For internal energy exchange, both the rotational and vibrational collision numbers have been fixed at 1 . The dsmcFoam Q-K results are compared with the analytical solution provided by Eq. (27). The forward rate coefficient $k_{f}(T)$ for Eq. (27) is provided as a best-fit Arrhenius curve to the equilibrium analytical Q-K data, where the Arrhenius rate takes the form

$$
k_{f}(T)=C_{1} T^{\left(C_{2}\right)} \exp \left(\frac{-E_{a}}{k T}\right)
$$

where $k$ is the Boltzmann constant, $C_{1}$ and $C_{2}$ are reaction-specific constants, and $E_{a}$ is the activation energy, the values of which are provided in Table $\underline{2}$.
Figures $\underline{8}$ and $\underline{9}$ show the evolution of species and overall temperature, respectively. The species concentrations at any instant in time $\left(n_{s}^{t}\right)$ have been nondimensionalized with respect to the initial number density of the species $\left(n^{o}\right)$. In general, the Q-K predictions of the constituent evolution agree well with the analytical result, although there is an underprediction of NO toward the end of the reactions. Oxygen scatter toward the tail of the reaction is due to its rapid depletion as the reactions progress. Considering the overall temperature field for the gas mixture, very good agreement between the DSMC and analytical solutions is evident for each case, with any scatter being explained by the extinction of the $\mathrm{O}_{2}$ and $\mathrm{NO}$ molecules toward the end of the reaction process.

These results highlight the important role that endothermic dissociation plays in acting as an energy sink in hypersonic reacting flows. The energy redistribution during the chemical reaction manifests itself as a reduction in the enthalpy of the flow, with a consequent reduction in surface heat transfer to the hypersonic body.

\section{Hypersonic Flow Test Case}

To test the dsmcFoam implementation of the Q-K chemistry model in a practical scenario a hypersonic flow test case is simulated. This consists of high-speed, rarefied air flow over a two-dimensional (2D) cylinder at the atmospheric freestream conditions shown in Table $\underline{3}$.

These conditions correspond to the Earth's atmosphere at $86 \mathrm{~km}$ altitude and a Mach number of 24.85. The cylinder walls are fully diffuse, and all 19 chemical reactions shown in Table 2 are activated. Comparisons are made with the equivalent solution using the established DSMC code MONACO, which employs TCE chemistry. In all cases, the rotational and vibrational collision numbers are set to 5 and 50, respectively, and the TCE Arrhenius rates for dissociation and exchange are provided from the $\mathrm{Q}-\mathrm{K}$ analytical equilibrium values, using Eq. (9) for dissociation and Eqs. (31) and (32) for exchange. The mesh size for the dsmcFoam study is 240,000 cells with a time step fixed at $1 \times 10^{-7} \mathrm{~s}$, and following particle sensitivity trials, there were 23.4 million DSMC particles in the system at steady state. For the MONACO study, the domain contains 175,000 cells with automatic subcell generation, and the total number of DSMC particles at steady state is 37.5 million. In addition, cell-based, variable time steps are used such that the ratio of the time step to mean collision time in each cell is approximately 0.2 . We suggest that this test case be considered as a reference $2-\mathrm{D}$ benchmark trial for future DSMC chemistry implementations.

Comparative tests between dsmcFoam and MONACO for nonreacting conditions showed excellent agreement between the aeorthermodynamic quantities as demonstrated in Fig. 10, which

Table 2 Chemical reaction list and equivalent Arrhenius rates

\begin{tabular}{lccccc}
\hline \hline Number & Reaction & $\begin{array}{c}\text { Heat of formation } \\
\left(\times 10^{19}\right), \mathrm{J}\end{array}$ & \multicolumn{1}{c}{$C_{1}$} & \multicolumn{1}{c}{$C_{2}$} & $\begin{array}{c}\text { Activation energy } \\
E_{a}\left(\times 10^{19}\right), \mathrm{J}\end{array}$ \\
\hline 1 & $\mathrm{O}_{2}+\mathrm{N} \rightarrow \mathrm{O}+\mathrm{O}+\mathrm{N}$ & 8.197 & $1.1 \times 10^{-10}$ & -1.0 & 8.197 \\
2 & $\mathrm{O}_{2}+\mathrm{NO} \rightarrow \mathrm{O}+\mathrm{O}+\mathrm{NO}$ & 8.197 & $1.1 \times 10^{-10}$ & -1.0 & 8.197 \\
3 & $\mathrm{O}_{2}+\mathrm{N}_{2} \rightarrow \mathrm{O}+\mathrm{O}+\mathrm{N}_{2}$ & 8.197 & $1.3 \times 10^{-10}$ & -1.0 & 8.197 \\
4 & $\mathrm{O}_{2}+\mathrm{O}_{2} \rightarrow \mathrm{O}+\mathrm{O}+\mathrm{O}_{2}$ & 8.197 & $5.33 \times 10^{-11}$ & -1.0 & 8.197 \\
5 & $\mathrm{O}_{2}+\mathrm{O} \rightarrow \mathrm{O}+\mathrm{O}+\mathrm{O}$ & 8.197 & $1.5 \times 10^{-10}$ & -1.05 & 8.197 \\
6 & $\mathrm{~N}_{2}+\mathrm{O} \rightarrow \mathrm{N}+\mathrm{N}+\mathrm{O}$ & 15.67 & $4.0 \times 10^{-12}$ & -0.54 & 15.67 \\
7 & $\mathrm{~N}_{2}+\mathrm{O}_{2} \rightarrow \mathrm{N}+\mathrm{N}+\mathrm{O}_{2}$ & 15.67 & $1.5 \times 10^{-11}$ & -0.68 & 15.67 \\
8 & $\mathrm{~N}_{2}+\mathrm{NO} \rightarrow \mathrm{N}+\mathrm{N}+\mathrm{NO}$ & 15.67 & $1.5 \times 10^{-11}$ & -0.68 & 15.67 \\
9 & $\mathrm{~N}_{2}+\mathrm{N}_{2} \rightarrow \mathrm{N}+\mathrm{N}+\mathrm{N}_{2}$ & 15.67 & $4.1 \times 10^{-12}$ & -0.62 & 15.67 \\
10 & $\mathrm{~N}_{2}+\mathrm{N} \rightarrow \mathrm{N}+\mathrm{N}+\mathrm{N}$ & 15.67 & $1.0 \times 10^{-11}$ & -0.68 & 15.67 \\
11 & $\mathrm{NO}+\mathrm{N} \rightarrow \mathrm{N}+\mathrm{O}+\mathrm{N}_{2}$ & 10.43 & $2.1 \times 10^{-10}$ & -1.0 & 10.43 \\
12 & $\mathrm{NO}+\mathrm{O} \rightarrow \mathrm{N}+\mathrm{O}+\mathrm{O}_{2}$ & 10.43 & $2.0 \times 10^{-10}$ & -1.0 & 10.43 \\
13 & $\mathrm{NO}+\mathrm{NO} \rightarrow \mathrm{N}+\mathrm{O}+\mathrm{NO}$ & 10.43 & $1.0 \times 10^{-10}$ & -1.0 & 10.43 \\
14 & $\mathrm{NO}+\mathrm{O} \rightarrow \mathrm{N}+\mathrm{O}+\mathrm{O}$ & 10.43 & $4.0 \times 10^{-10}$ & -1.1 & 10.43 \\
15 & $\mathrm{NO}+\mathrm{N} \rightarrow \mathrm{N}+\mathrm{O}+\mathrm{N}$ & 10.43 & $4.0 \times 10^{-10}$ & -1.1 & 10.43 \\
16 & $\mathrm{NO}+\mathrm{O} \rightarrow \mathrm{O}+\mathrm{N}$ & 2.719 & $2.3 \times 10^{-19}$ & 0.50 & 2.719 \\
17 & $\mathrm{~N}_{2}+\mathrm{O} \rightarrow \mathrm{NO}+\mathrm{N}$ & 5.175 & $0.8 \times 10^{-16}$ & 0 & 5.175 \\
18 & $\mathrm{O}+\mathrm{N} \rightarrow \mathrm{NO}+\mathrm{O}$ & -2.719 & $4.0 \times 10^{-15}$ & -0.39 & 0.2 \\
19 & $\mathrm{NO}+\mathrm{N} \rightarrow \mathrm{N}+\mathrm{O}$ & -5.175 & $5.0 \times 10^{-16}$ & -0.35 & 0.2 \\
\hline \hline
\end{tabular}




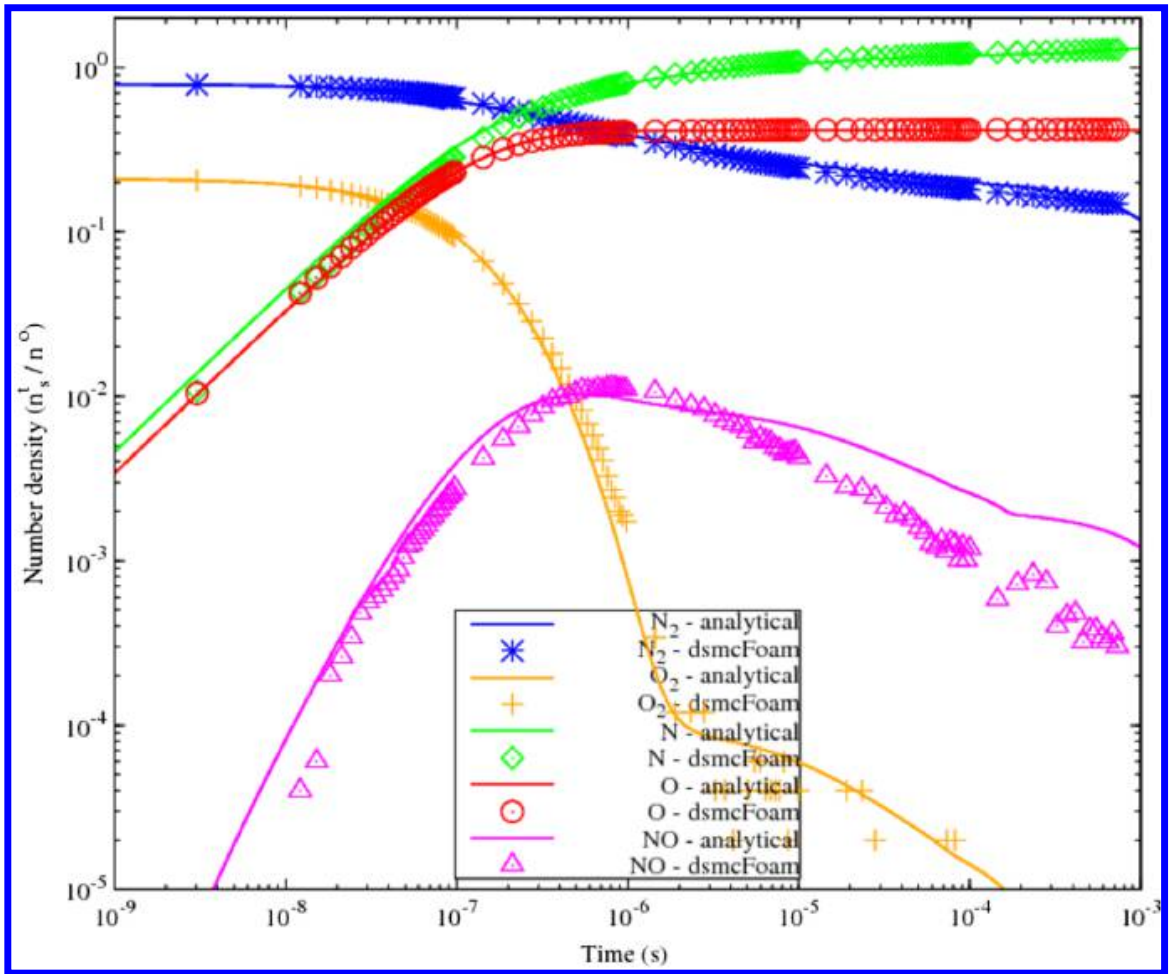

Fig. 8 Species concentrations during the decomposition of air from an initial temperature of 30,000 K and pressure of 0.063 atm.

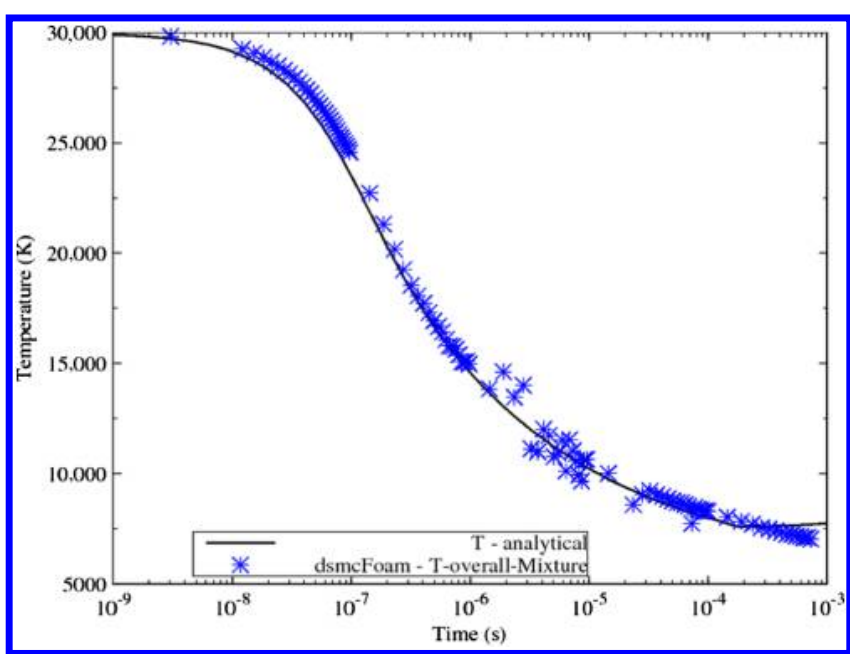

Fig. 9 Overall temperature evolution of the gas mixture during the decomposition of air from an initial temperature of $30,000 \mathrm{~K}$ and pressure of $0.063 \mathrm{~atm}$.

shows the temperature profile along the stagnation streamline. Figures 11-13 show the reacting flow comparisons between the DSMC codes for velocity, density, and temperature, respectively,

Table 3 Freestream conditions for 2-D hypersonic test case

\begin{tabular}{lc}
\hline \hline Parameter & Value \\
\hline Cylinder diameter & $2 \mathrm{~m}$ \\
Flow velocity & $6813 \mathrm{~m} / \mathrm{s}$ \\
Freestream temperature & $187 \mathrm{~K}$ \\
Cylinder wall temperature & $1000 \mathrm{~K}$ \\
$\mathrm{~N}_{2}$ number density & $1.13 \times 10^{20} \mathrm{~m}^{-3}$ \\
$\mathrm{O}_{2}$ number density & $3.031 \times 10^{19} \mathrm{~m}^{-3}$ \\
\hline \hline
\end{tabular}

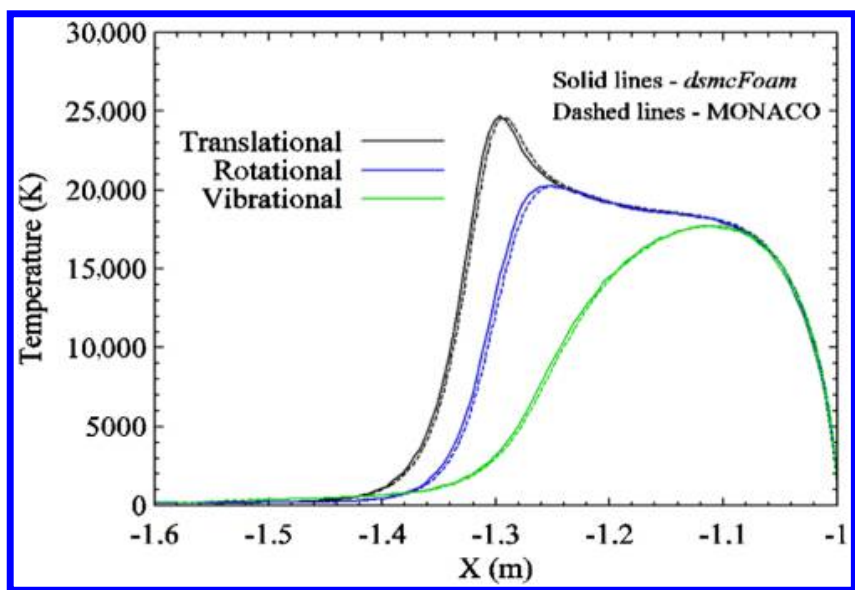

Fig. 10 Temperature profile along the stagnation streamline for nonreacting air flow over a 2-D cylinder.

along the stagnation streamline. Because the chemical reactions that occur are mainly characterized by endothermicity, the local enthalpy is reduced in comparison to an inert flow, and the maximum shock standoff distance is located at a position approximately $0.36 \mathrm{~m}$ from the cylinder stagnation point.

Figure 12 shows that the stagnation streamline densities for $\mathrm{N}_{2}$ and NO calculated by each code are in relatively close agreement. Although the trends are similar, the values for the atomic species $\mathrm{N}$ and O predicted with dsmcFoam (Q-K) are in excess of those determined by MONACO (TCE). However, the opposite is true for $\mathrm{O}_{2}$, with the MONACO (TCE) code producing values exceeding those of dsmcFoam $(\mathrm{Q}-\mathrm{K})$. The increased number of dissociation events when using Q-K may be qualitatively explained with reference to Wysong et al. [19]: the magnitude of the lower level vibrational cross sections for the Q-K method would mean that more dissociation events are likely, compared with the TCE approach, in nonequilibrium flows with relatively low-vibrational excitation, such as high-altitude reentry. 


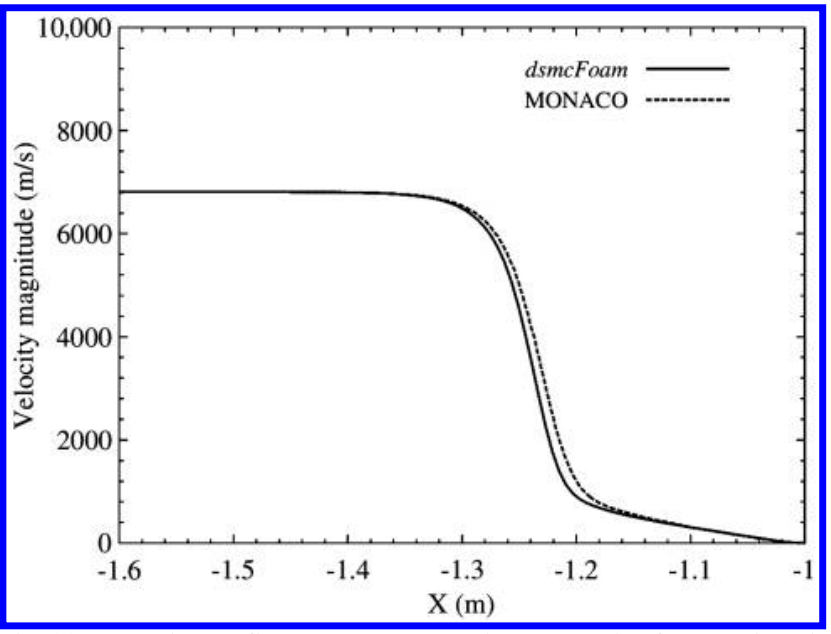

Fig. 11 Velocity profile along the stagnation streamline for reacting air flow over a 2-D cylinder.

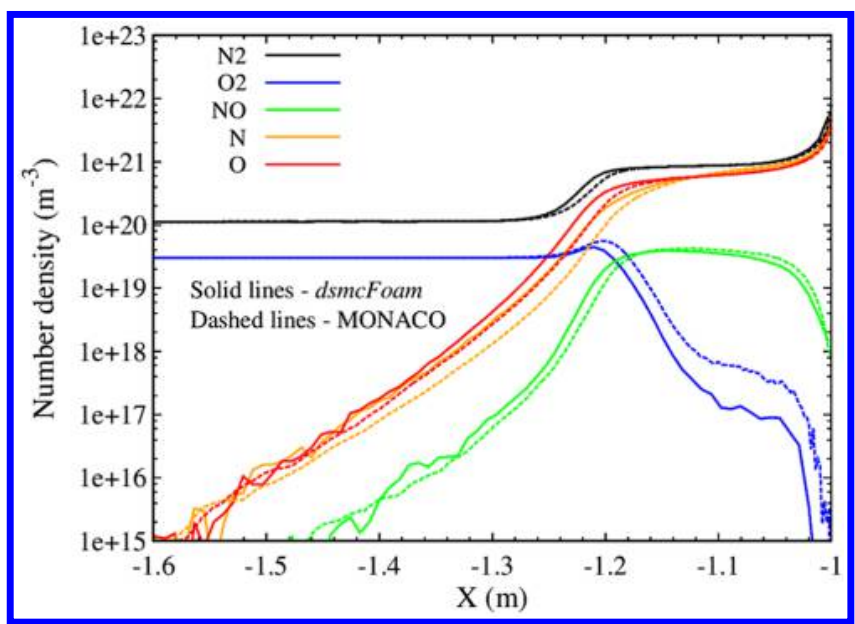

Fig. 12 Number density profile along the stagnation streamline for reacting air flow over a $2-\mathrm{D}$ cylinder.

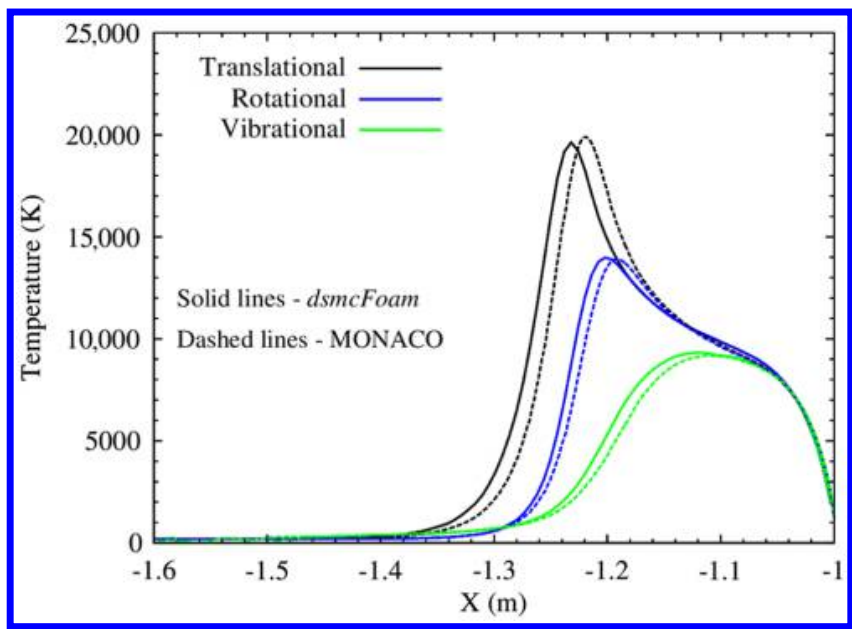

Fig. 13 Temperature profiles along the stagnation streamline for reacting air flow over a 2 -D cylinder.

Significant reductions in the values of all three temperature modes under reacting flow conditions are evident in Fig. 13, with both codes predicting a peak translational temperature along the stagnation streamline of approximately $20,000 \mathrm{~K}$. This is in contrast to the non-

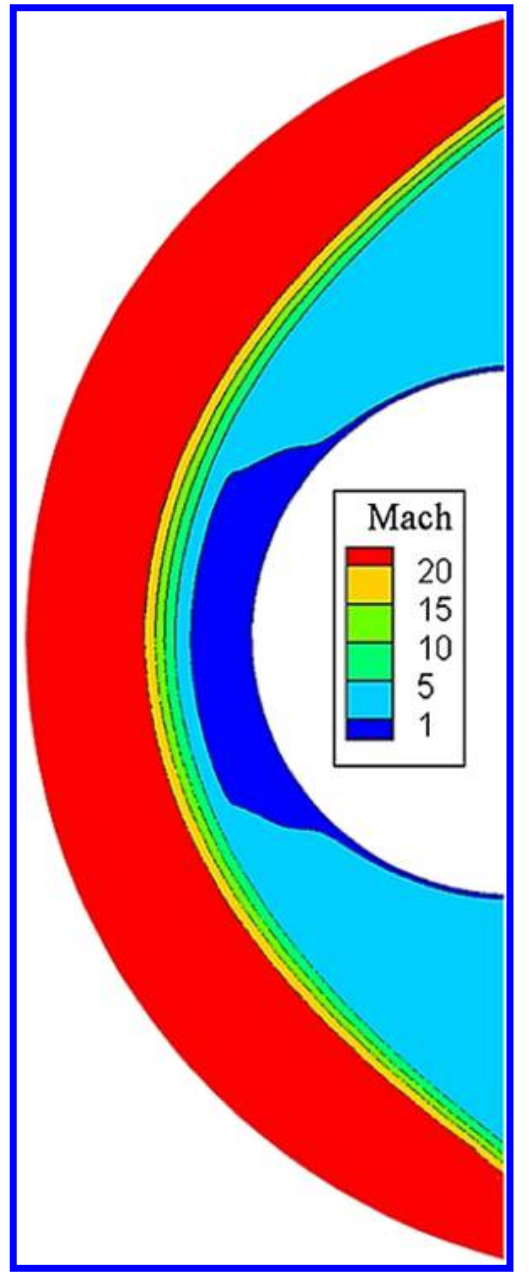

Fig. 14 Mach-number contours predicted using dsmcFoam (Q-K, lower half) and MONACO (TCE, upper half) for reacting air flow over a 2-D cylinder.

reacting case (Fig. 10), for which the peak translational magnitude is approximately $25,000 \mathrm{~K}$. The peak values for rotational and vibrational temperature are also in close agreement; however, the general dsmcFoam Q-K temperature values show a small but consistent shift to the left in comparison with the MONACO-based ones. This effect is consistent with the differences in the shock structure indicated by the leftward velocity shift shown in Fig. 11.

Figures 14 and 15 show the contours of Mach number and NO density for each code, respectively. It is clear that the diffuse nature of the shock has been captured by both codes. Although differences exist between the results of the codes for the predicted velocity and temperature fields, it is evident that the local Mach numbers are in close agreement. For the NO field, it is seen that small differences are evident between the solutions in this qualitative comparison, with the Q-K approach appearing to predict a marginally thinner species layer in the range approaching the peak NO values. Nonetheless, the general flow features appear to be similar for both chemistry approaches.

The translational temperature mode is shown in Fig. 16, and in general, a similar qualitative concurrence is evident in all areas of the flowfield, with only subtle differences apparent in certain regions.

Finally, the surface properties are shown in Figs. 17 and $\underline{18}$. The surface pressure values are in very close agreement between the codes. The peak-value of the heat flux is $64 \mathrm{~kW} / \mathrm{m}^{2}$ for TCE and $67 \mathrm{~kW} / \mathrm{m}^{2}$ for $\mathrm{Q}-\mathrm{K}$. This difference of $4.6 \%$ represents a similar margin to the range of peak heat fluxes found in a separate case study of nonreacting flow over a 2-D cylinder using a variety of DSMC codes [26]. Figures 19 and 20 show the surface temperature-jump and velocity-slip predictions. The general trends are similar for both 


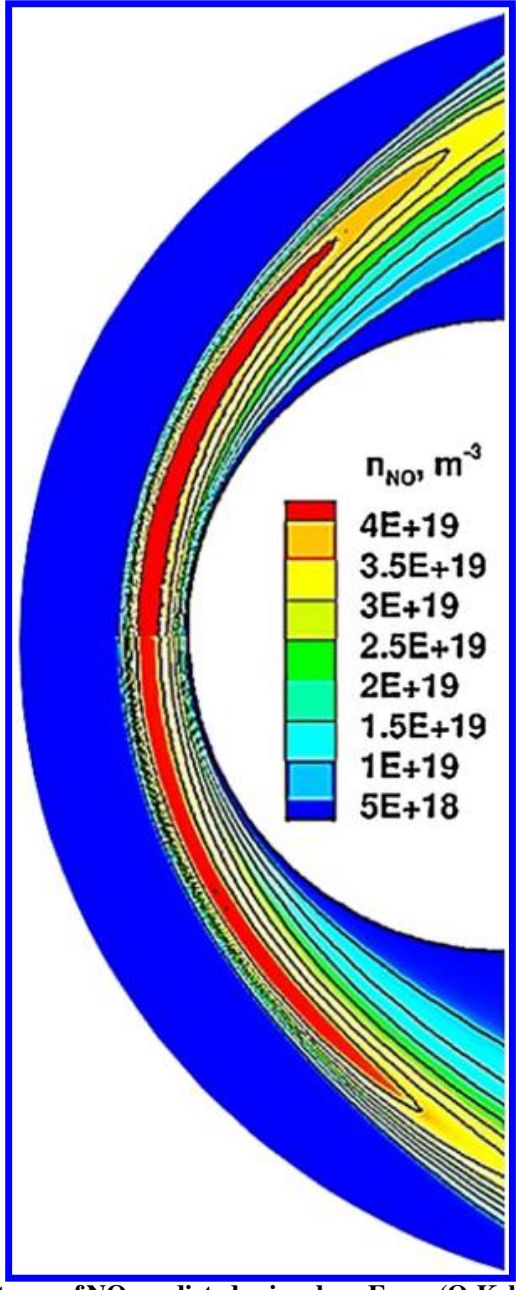

Fig. 15 Contours of NO predicted using dsmcFoam (Q-K, lower half) and MONACO (TCE, upper half) for reacting air flow over a 2-D cylinder.

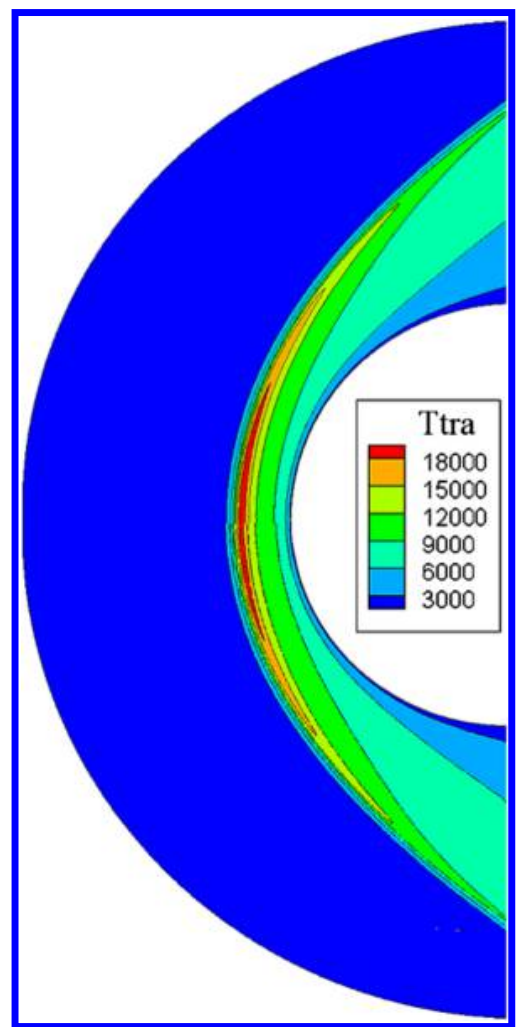

Fig. 16 Translational temperature contours predicted using dsmcFoam (Q-K, lower half) and MONACO (TCE, upper half) for reacting air flow over a 2-D cylinder.

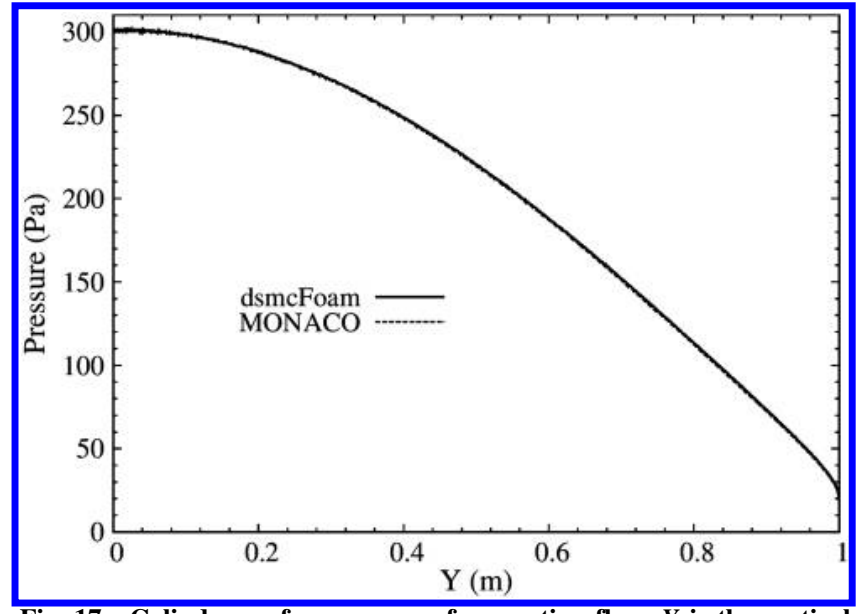

Fig. 17 Cylinder surface pressure for reacting flow. $Y$ is the vertica position above the stagnation point.

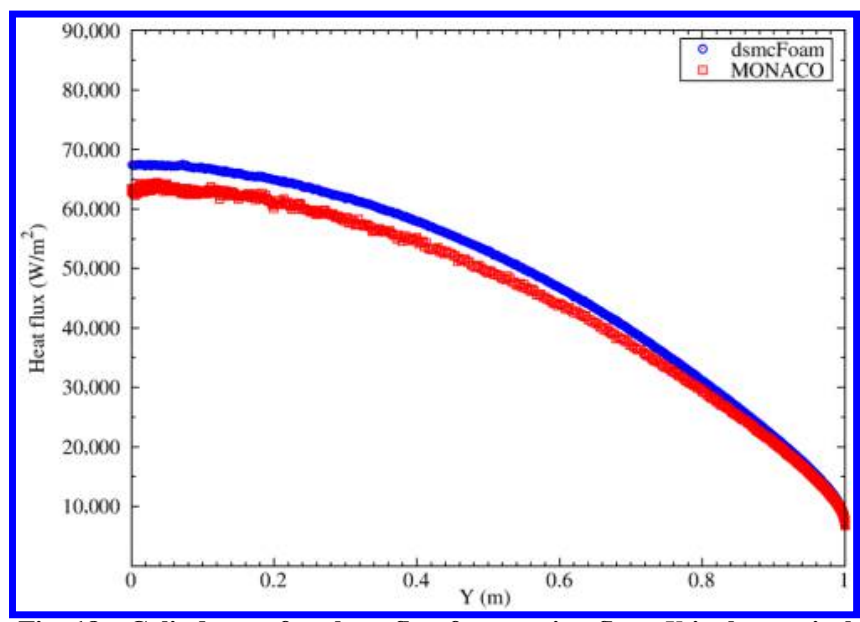

Fig. 18 Cylinder surface heat flux for reacting flow. $Y$ is the vertical position above the stagnation point.

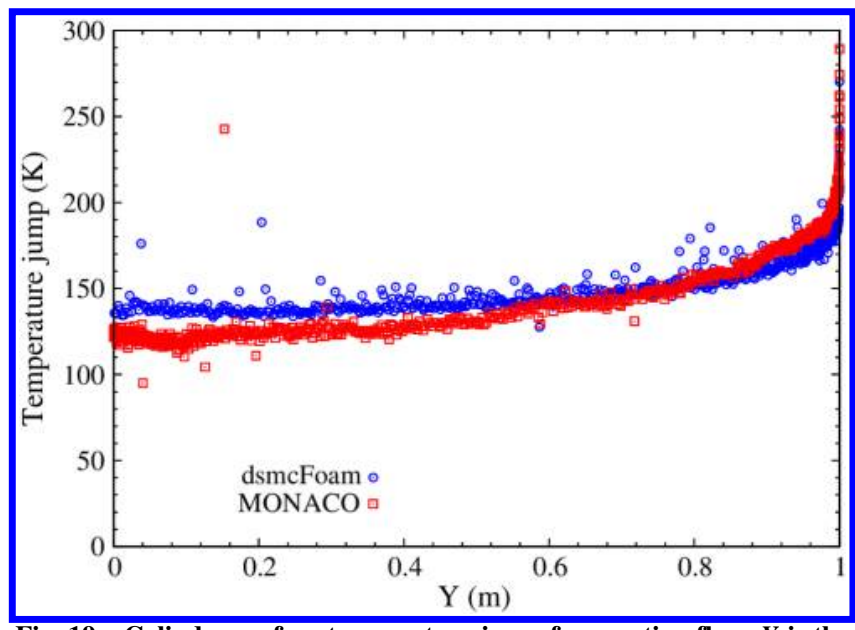

Fig. 19 Cylinder surface temperature jump for reacting flow. $Y$ is the vertical position above the stagnation point.

MONACO and dsmcFoam; however, there appears to be a greater level of disparity at some locations on the cylinder surface compared with the results for pressure and heat flux, with dsmcFoam predicting generally higher values of slip and jump. 


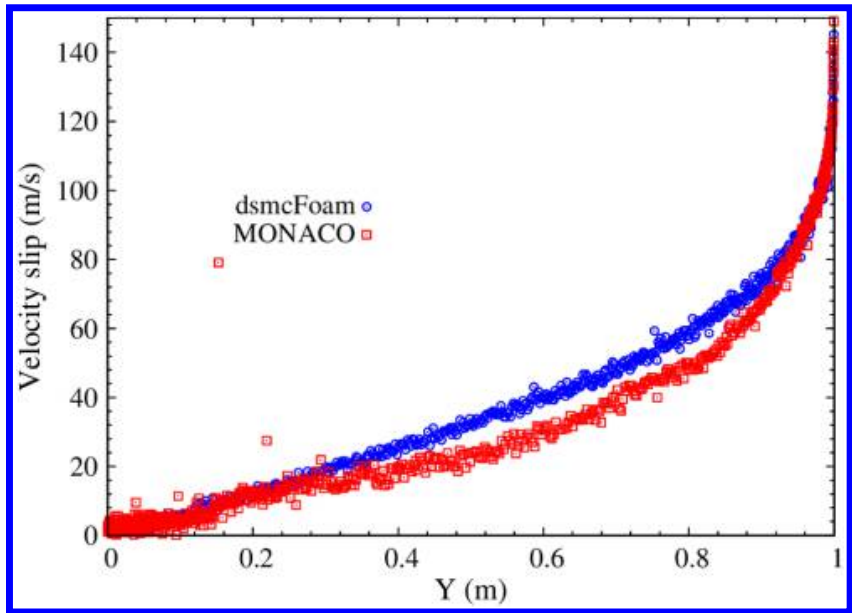

Fig. 20 Cylinder surface velocity slip for reacting flow. $Y$ is the vertical position above the stagnation point.

\section{Conclusions}

An open-source DSMC chemistry model based on the Q-K approach has been developed for use in high-speed, rarefied gas flows. The code, called dsmcFoam, has been written within the framework of the open-source CFD software suite OpenFOAM. Benchmark test cases demonstrate the successful implementation of the Q-K code into dsmcFoam, and comparisons with analytical results for dissociation and exchange reactions showed a satisfactory degree of concurrence. Differences in the predicted equilibrium rates for Q-K exchange have been highlighted when macroscopic and collision temperatures are used. New air reaction rates for exchange have been determined, based on the Q-K approach, and presented in Arrhenius form. A test case was run for hypersonic crossflow over a 2-D cylinder, and the dsmcFoam (Q-K) results were found to be in broad agreement with the established DSMC code MONACO, which employs TCE procedures for its reaction modeling. Differences in predicted species profiles along the stagnation streamline for Q-K and TCE have been qualitatively explained. This hypersonic test case is suggested as a reference 2-D benchmark trial for future DSMC chemistry implementations.

\section{Acknowledgments}

Thomas Scanlon wishes to acknowledge the support provided by the Engineering and Physical Sciences Research Council (EPSRC) grant number EP/J005924/1. International Collaboration Sabbatical. dsmcFoam results were obtained using the EPSRC-funded ARCHIEWeSt High Performance Computer (http://www.archie-west.ac.uk), EPSRC grant number EP/K000586/1. The work at the University of Michigan is funded through grants FA9550-11-1-0309 and FA955012-1-0483 from the U.S. Air Force Office of Scientific Research. Jason Reese and Matthew Borg wish to acknowledge the support provided by the EPSRC grant EP/I011927/1.

\section{References}

[1] Gallis, M. A., Bond, R. B., and Torczynski, J. R., "A Kinetic-Theory Approach for Computing Chemical-Reaction Rates in UpperAtmosphere Hypersonic Flows," Journal of Chemical Physics, Vol. 131, No. 12, 2009, Paper 124311 doi:10.1063/1.3241133

-[2] Bertin, J. J., and Cummings, R. M., "Critical Hypersonic Aerothermodynamic Phenomena," Annual Review of Fluid Mechanics, Vol. 38, Jan. 2006, pp. 129-157.

doi:10.1146/annurev.fluid.38.050304.092041

-[3] Bhatnagar, P. L., Gross, E. P., and Krook, M., "A Model Collision Process in Gases," Physical Review, Vol. 94, May 1954, pp. 511-525. doi:10.1103/PhysRev.94.511

- [4] Yang, J. Y., and Huang, J. C., "Rarefied Flow Computations Using Non-Linear Model Boltzmann Equations," Journal of Computational
Physics, Vol. 120, No. 2, 1995, pp. 323-339.

doi:10.1006/jcph.1995.1168

-[5] Greenshields, C. J., and Reese, J. M., "The Structure of Shock Waves as a Test of Brenner's Modifications to the Navier-Stokes Equations," Journal of Fluid Mechanics, Vol. 580, June 2007, pp. 407-429. doi:10.1017/S0022112007005575

[6] Bird, G. A., Molecular Gas Dynamics and the Direct Simulation of Gas Flows, Clarendon, Oxford, 1994.

[7] Wagner, W., "A Convergence Proof for Bird's Direct Simulation Monte Carlo Method for the Boltzmann Equation," Journal of Statistical Physics, Vol. 66, Nos. 3-4, 1992, pp. 1011-1044. doi:10.1007/BF01055714

[8] Anderson, J. D., Jr., Hypersonic and High Temperature Gas Dynamics, 2nd ed., McGraw-Hill, New York, 2006.

- [9] Bird, G. A., "The Q-K Model for Gas Phase Chemical Reaction Rates," Physics of Fluids, Vol. 23, No. 10, 2011, Paper 106101. doi: $10.1063 / 1.3650424$

[10] OpenCFD Ltd., Software Package, Ver. 2.3, Bracknell, England, U.K., www.openfoam.com [retrieved 26 Feb. 2015].

-[11] Scanlon, T. J., Roohi, E., White, C., Darbandi, M., and Reese, J. M., "An Open Source, Parallel, DSMC Code for Rarefied Gas Flows in Arbitrary Geometries," Computers \& Fluids, Vol. 39, No. 10, 2010, pp. 20782089. doi:10.1016/j.compfluid.2010.07.014

[12] Haas, B. L., McDonald, J. D., and Dagum, L., "Models of Thermal Relaxation Mechanics for Particle Simulations," Journal of Computational Physics, Vol. 107, No. 2, 1993, pp. 348-358. doi:10.1006/jcph.1993.1149

[13] Bergemann, F., and Boyd, I. D., "New Discrete Vibrational Energy Model for the Direct Simulation Monte Carlo Method," Progress in Astronautics and Aeronautics, Vol. 158, 1994, pp. 174-183.

[14] Bird, G. A., "A Comparison of Collision Energy-Based and Temperature-Based Procedures in DSMC," AIP Conference Proceedings, Vol. 1084, No. 1, 2008, pp. 245-250.

[15] Zuppardi, G., Morsa, L., and Romano, F., "Influence of Chemical Models on the Computation of Thermo-Fluid-Dynamic Parameters in Hypersonic, Rarefied Flows," Proceedings of the Institution of Mechanical Engineers, Part G, Vol. 224, No. 6, 2010, pp. 637-646. doi:10.1243/09544100JAERO713

[16] Bird, G. A., "Sophisticated DSMC," Notes Prepared for Short Course at the DSMC07 Meeting, Santa Fe, NM, 2007, p. 31, http://www.gab.com .au/dsmc07notes.pdf [retrieved 26 Feb. 2015]

[17] Bird, G. A., "Simulation of Multi-Dimensional and Chemically Reacting Flows (Past Space Shuttle Orbiter)," Rarefied Gas Dynamics, Vol. 1, edited by Campargue, R., Commissariat a L'energie Atomique, Paris, 1979, pp. 365-388.

[18] Boyd, I. D., "Modeling Backward Chemical Rate Processes in the Direct Simulation Monte Carlo Method," Physics of Fluids, Vol. 19, No. 12 2007, Paper 126103. doi:10.1063/1.2825038

[19] Wysong, I., Gimelshein, S., Gimelshein, N., McKeon, W., and Esposito, F., "Reaction Cross Sections for Two Direct Simulation Monte Carlo Models: Accuracy and Sensitivity Analysis," Physics of Fluids, Vol. 24, No. 4, 2012, Paper 042002. doi:10.1063/1.3701379

[20] Vincenti, W. G., and Kruger, C. H., Introduction to Physical Gas Dynamics, Wiley, New York, 1965.

-[21] Haas, B. L., and McDonald, J. D., "Validation of Chemistry Models Employed in a Particle Simulation Method," Journal of Thermophysics and Heat Transfer, Vol. 7, No. 1, 1993, pp. 42-48. doi: $10.2514 / 3.11567$

[22] Bird, G. A., "DSMC Resources from Graeme Bird," 2014, http://www .gab.com.au [retrieved 26 Feb. 2015].

[23] National Inst. for Standards and Technology, Gaithersburg, MD, http:// webbook.nist.gov/chemistry/ [retrieved 26 Feb. 2015].

[24] Gupta, R. N., Yos, J. M., Thompson, R. A., and Lee, K. P., "A Review of Reaction Rates and Thermodynamics and Transport Properties from an 11-Species Air Model for Chemical and Thermal Non-Equilibrium Calculation to 30,000 K," NASA Reference Publ. 1232, Aug. 1990.

[25] Bortner, M. H., "A Review of Rate Constants of Selected Reactions of Interest in Re-Entry Flow Fields in Atmosphere," U.S. Department of Commerce, Rept. NBS TN-484, Washington, D.C., 1966.

[26] Bird, G. A., The DSMC Method, Ver. 1.1, Aug. 2013, p. 225, https:// www.createspace.com/3689652 [retrieved 26 Feb. 2015] 
This article has been cited by:

1. C. White, M.K. Borg, T.J. Scanlon, S.M. Longshaw, B. John, D.R. Emerson, J.M. Reese. 2017. dsmcFoam+: An OpenFOAM based direct simulation Monte Carlo solver. Computer Physics Communications . [Crossref]

2. Rodrigo C. Palharini, João Luiz F. Azevedo. 2017. Thermochemical Nonequilibrium Computations of a Brazilian Reentry Satellite. Journal of Spacecraft and Rockets 54:4, 961-966. [Citation] [Full Text] [PDF] [PDF Plus]

3. Ramin Zakeri, Ramin Kamali Moghadam, Mahmoud Mani. 2017. New chemical-DSMC method in numerical simulation of axisymmetric rarefied reactive flow. Physics of Fluids 29:4, 047105. [Crossref]

4. Tobias Baier, Steffen Hardt, Vahid Shahabi, Ehsan Roohi. 2017. Knudsen pump inspired by Crookes radiometer with a specular wall. Physical Review Fluids 2:3. . [Crossref]

5. Amin Ebrahimi, Ehsan Roohi. 2017. DSMC investigation of rarefied gas flow through diverging micro- and nanochannels. Microfluidics and Nanofluidics 21:2. . [Crossref]

6. V. A. Istomin, E. V. Kustova. 2017. Transport coefficients and heat fluxes in non-equilibrium high-temperature flows with electronic excitation. Physics of Plasmas 24:2, 022109. [Crossref]

7. O. V. Kunova, G. V. Shoev, A. N. Kudryavtsev. 2017. Numerical simulation of nonequilibrium flows by using the stateto-state approach in commercial software. Thermophysics and Aeromechanics 24:1, 7-17. [Crossref]

8. Vincent Casseau, Rodrigo Palharini, Thomas Scanlon, Richard Brown. 2016. A Two-Temperature Open-Source CFD Model for Hypersonic Reacting Flows, Part One: Zero-Dimensional Analysis. Aerospace 3:4, 34. [Crossref]

9. Vincent Casseau, Daniel Espinoza, Thomas Scanlon, Richard Brown. 2016. A Two-Temperature Open-Source CFD Model for Hypersonic Reacting Flows, Part Two: Multi-Dimensional Analysis †. Aerospace 3:4, 45. [Crossref]

10. Ramin Zakeri, Ramin Kamali-Moghadam, Mahmoud Mani. 2016. A new approach for chemical reaction simulation of rarefied gas flow by DSMC method. Computers \& Fluids 140, 111-121. [Crossref]

11. Chariton Christou, S. Kokou Dadzie. 2016. Direct-Simulation Monte Carlo Investigation of a Berea Porous Structure. SPE Journal 21:03, 0938-0946. [Crossref]

12. Benzi John, Xiao-Jun Gu, Robert W. Barber, David R. Emerson. 2016. High-Speed Rarefied Flow Past a Rotating Cylinder: The Inverse Magnus Effect. ALAA Journal 54:5, 1670-1681. [Abstract] [Full Text] [PDF] [PDF Plus]

13. V. A. Istomin, E. V. Kustova. Effect of electronic excitation on high-temperature flows of ionized nitrogen and oxygen mixtures behind strong shock waves 150003. [Crossref]

14. D. E. R. Espinoza, V. Casseau, T. J. Scanlon, R. E. Brown. An open-source hybrid CFD-DSMC solver for high speed flows 050007. [Crossref]

15. Benzi John, Xiao-Jun Gu, Robert W. Barber, David R. Emerson. On the inverse Magnus effect for flow past a rotating cylinder 050022. [Crossref]

16. V. Casseau, T. J. Scanlon, B. John, D. R. Emerson, R. E. Brown. Hypersonic simulations using open-source CFD and DSMC solvers 050006. [Crossref]

17. Stephen Voelkel, Damien Masselot, Philip L. Varghese, Venkat Raman. Analysis of hydrogen-air detonation waves with vibrational nonequilibrium 070015. [Crossref]

18. O. Kunova, E. Nagnibeda, I. Sharafutdinov. Non-equilibrium reaction rates in air flows behind shock waves. State-to-state and three-temperature description 150005. [Crossref]

19. C. White, T. J. Scanlon, J. A. Merrifield, K. Kontis, T. Langener, J. Alves. Numerical and experimental capabilities for studying rocket plume-regolith interactions 170003. [Crossref]

20. E. Kustova, E. Nagnibeda, G. Oblapenko, A. Savelev, I. Sharafutdinov. 2016. Advanced models for vibrational-chemical coupling in multi-temperature flows. Chemical Physics 464, 1-13. [Crossref]

21. Rodrigo C. Palharini, Craig White, Thomas J. Scanlon, Richard E. Brown, Matthew K. Borg, Jason M. Reese. 2015. Benchmark numerical simulations of rarefied non-reacting gas flows using an open-source DSMC code. Computers \& Fluids 120, 140-157. [Crossref]

22. Vincent Casseau, Thomas J Scanlon, Richard E. Brown. Development of a Two-Temperature Open-Source CFD Model for Hypersonic Reacting Flows . [Citation] [PDF] [PDF Plus]

23. Rodrigo C. Palharini, Joao Luiz F. Azevedo. Thermochemical Nonequilibrium Computations of a Brazilian Reentry Satellite . [Citation] [PDF] [PDF Plus] 